\title{
Ordering of caspases in cells undergoing apoptosis by the intrinsic pathway
}

\author{
S Inoue ${ }^{1}$, G Browne ${ }^{1}$, G Melino ${ }^{1}$ and GM Cohen ${ }^{\star, 1}$
}

Caspases are a family of aspartate-specific cysteine proteases responsible for the biochemical and morphological changes that occur during the execution phase of apoptosis. The hierarchical ordering of caspases has been clearly established using dATPactivated cell lysates to model the intrinsic pathway induced by initial mitochondrial perturbation. In this model, caspase-9, the apical caspase, directly processes and activates the effector caspases, caspase- 3 and -7 , and then active caspase- 3 but not caspase-7, processes caspase -2 and -6 , and subsequently the activated caspase- 6 processes caspase -8 and -10 . To address the possibility that this model in vitro system might not reflect the precise ordering of caspases in intact cells, we have examined this possibility in cells induced to undergo apoptosis by activation of the intrinsic pathway. We have used caspase deficient cells, small interference RNA for caspase- 6 and -7 , and a specific caspase- 3 inhibitor. In contrast to the earlier in vitro studies, we now show that in intact cells caspase- 7 can also directly process and activate caspase-2 and -6 . The processing of caspase-2 and -6 occurs within the cytoplasm and active caspase- 6 is then responsible for both the processing of caspase- 8 and the cleavage of caspase- 6 substrates, including lamin A/C.

Cell Death and Differentiation (2009) 16, 1053-1061; doi:10.1038/cdd.2009.29; published online 27 March 2009

Apoptosis, a major form of cell death used to remove unwanted or excess cells, is characterized by a plethora of morphological and biochemical changes resulting primarily from the activation of a family of cysteine proteases, which cleave their substrates at specific aspartate residues. ${ }^{1-4}$ Two major apoptotic pathways have been described, the intrinsic pathway involving initial mitochondrial perturbation resulting from cellular stress or cytotoxic insults and the extrinsic pathway, which is triggered by the activation of death receptors of the TNF family. ${ }^{5-7}$ Caspase-8 and -9 , the apical caspases in the extrinsic and intrinsic pathways, possess a protein interaction prodomain, which result in their recruitment and subsequent activation within cellular complexes, namely the death-inducing signaling complex (DISC) and the Apaf-1 apoptosome, respectively, and the subsequent activation of effector caspases, such as caspase-3, -6 and $-7 . .^{5,8-10}$ Caspases are synthesized as single-chain zymogens, containing an $\mathrm{N}$-terminal prodomain, as well as large $(\sim 20 \mathrm{kDa})$ and small $(\sim 10 \mathrm{kDa})$ subunits, with the basic catalytic domain forming from a large and small subunits. ${ }^{1,3,4}$ Caspase-2 also has a sizeable prodomain, but it is unclear whether it should be considered an initiator or an effector caspase. ${ }^{3}$ Although caspase-2 has been proposed to act upstream of mitochondria and to be activated within the PIDDosome, it may also be activated downstream of caspase- $3 .{ }^{11-14}$ Using recombinant proteins, caspase-3, -8 and to a lesser extent caspase-7 can process caspase-2. ${ }^{15}$ The physiological role of caspase- 2 is not known and no marked phenotype is observed in caspase- $2^{-l-}$ mice. Caspase- 6 is generally considered to be an effector caspase. Caspase- 6 cleaves several substrates including nuclear lamins, focal adhesion kinase, nuclear mitotic apparatus protein (NUMA) and the nuclear matrix protein, special AT-rich binding protein-1 (SATB1). ${ }^{16-19}$ Most caspase- 6 substrates, except for lamin A/C and SATB1, can be cleaved by other caspases. In the absence of caspase-6, cells with lamin A do not undergo complete nuclear condensation during the execution phase of apoptosis. ${ }^{20}$ In the intrinsic pathway, mitochondrial perturbation results in the release of cytochrome $c$ to the cytosol, which in conjunction with dATP/ATP, causes Apaf-1 oligomerization and caspase9 activation. Caspase- 9 bound within the apoptosome complex to Apaf-1 through their CARD (caspase activation and recruitment domain) domains is $2-3$ orders of magnitude more active than free caspase- 9 within the cytosol. ${ }^{21}$ Within the apoptosome, caspase- 9 processes and activates both caspase- 3 and -7 by cleaving between their large and small subunits. Although the initial sequential processing and activation of caspase- $9,-3$ and -7 is generally well established, the precise hierarchical ordering of other caspases within the caspase cascade is less clear, particularly in cells undergoing apoptosis. A hierarchy of caspase activation was established using a model system of dATP-activated lysates to mimic events following activation of the intrinsic pathway and this paradigm is still believed to hold. ${ }^{18,22,23}$ In this system, caspase- 9 is the apical caspase that directly processes and activates caspase- 3 and -7 . Caspase- 3 but

\footnotetext{
${ }^{1}$ MRC Toxicology Unit, Hodgkin Building, University of Leicester, PO Box 138, Lancaster Road, Leicester LE1 9HN, UK

*Corresponding author: GM Cohen, MRC Toxicology Unit, University of Leicester, Hodgkin Building, PO Box 138, Lancaster Road Leicester, Leicestershire LE1 9HN, UK. Tel: + 44116252 5601; Fax: + 44116252 5616; E-mail: gmc2@le.ac.uk

Keywords: caspase; apoptosis; intrinsic pathway; MCF-7 cells; M-791

Abbreviations: PARP, poly(ADP-ribose)polymerase; PS, phosphatidylserine; TRAIL, TNF-related apoptosis-inducing ligand; XIAP, X-linked inhibitor of apoptosis; z-VAD.fmk, benzyloxycarbonyl-Val-Ala-Asp(OMe) fluoromethyl ketone

Received 17.12.08; revised 17.2.09; accepted 19.2.09; Edited by S Kaufmann; published online 27.3.09
} 
not caspase- 7 then processes caspase- 6 and -2 , and active caspase- 6 cleaves caspase- 8 and $-10 .^{18,22}$ Caspase- 3 was also shown to be the major effector caspase responsible for the cleavage of cellular substrates during apoptosis. However, in such an in vitro system, an active caspase may cleave other caspases but this does not necessarily reflect the cellular situation, where the amounts of enzymes and their compartmentation, may also be important. Although this model of caspase activation is currently widely accepted, ${ }^{23}$ there are some inconsistencies between the model and the phenotype from caspase- $3^{-/-}$, caspase $-7^{-1-}$ and caspase- $3^{-1-}$ /caspase- $7^{-1-}$ double-knockout mice. ${ }^{24}$ The double knockout mice died shortly after birth because of a defect in cardiac development and caspase-3 seemed to play a prominent role in DNA degradation, whereas caspase-3 and -7 were important in cell viability. ${ }^{24}$ Furthermore, caspase $-7^{-1-}$ mice had a relatively mild phenotype most likely because of compensation from caspase- 3 and both caspase-3 and -7 were key mediators of the loss of mitochondrial membrane potential. ${ }^{24}$ As these data suggested that the hierarchical ordering of caspases observed in dATPactivated cellular lysates may not truly reflect the situation in cells undergoing apoptosis, we set out to establish the precise ordering in a more relevant physiological scenario. We now show that caspase- 6 can be processed by caspase- 7 in a caspase-3 independent manner in cells undergoing apoptosis in contrast to the in vitro model system using dATP-activated lysates. Thus, our studies show an alternative pathway for the activation of caspase-6, -2 and -8 in intact cells under conditions, where caspase- 3 is either absent or selectively inactivated.

\section{Results}

Marked differences in caspase processing in Jurkat cells in the presence of Z-VAD.fmk and M-791. In the initial studies, Jurkat cells were exposed for $6 \mathrm{~h}$ to etoposide in the presence of z-VAD.fmk $(5-20 \mu \mathrm{M})$, a broad spectrum caspase inhibitor or M-791 (2.5-20 $\mu \mathrm{M})$, a specific caspase-3 inhibitor. ${ }^{25}$ Both inhibitors caused a concentration-dependent inhibition of apoptosis, with z-VAD.fmk being more potent (Figure 1). However, striking and subtle differences were observed in the processing of some caspases in the presence of the inhibitors. In untreated cells, caspase-2, -3 , $-6,-7,-8$ and -9 were present as their unprocessed zymogens (Figure 1, lane 1). Induction of apoptosis by etoposide occurs through the intrinsic pathway and was accompanied by the processing of all these caspases (Figure 1, lane 2). After activation by the Apaf- 1 apoptosome, caspase- 9 is initially cleaved at D315 to yield a p35 fragment. Within the apoptosome, caspase- 9 can then cleave and activate both caspase- 3 and -7 between their large and small subunits to yield their p20 and p17 large subunits, respectively. . $^{8,10}$ Active caspase- 3 can then cleave caspase- 9 at D330 to yield a p37 fragment. ${ }^{8,26}$ In agreement with these earlier studies, caspase-9 was cleaved at both D315 and 330 to yield the p35/37 forms (Figure 1a). Z-VAD.fmk and to a lesser extent M-791 caused a concentration-dependent inhibition of caspase-9 processing at D315, compatible with z-VAD.fmk

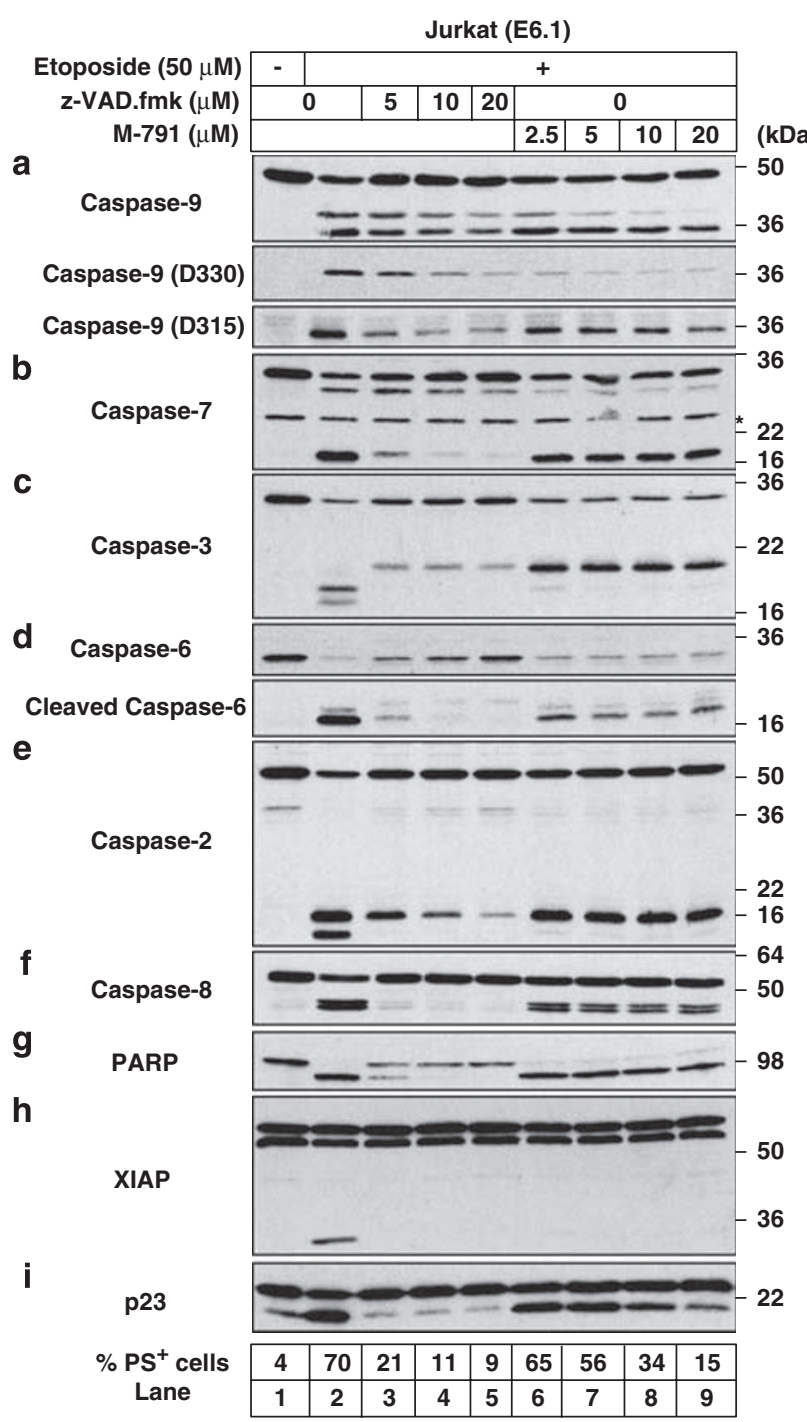

Figure 1 Differential inhibition of caspase processing in Jurkat cells. Jurkat cells (E6.1) were exposed for $6 \mathrm{~h}$ to etoposide $(50 \mu \mathrm{M})$ in the presence or absence of either z-VAD.fmk $(5-20 \mu \mathrm{M})$ or M-791 $(2.5-20 \mu \mathrm{M})$ as indicated. Apoptosis was assessed by PS externalization as described in Materials and Methods and cells were analyzed by western blotting either in $(\mathrm{a}-\mathrm{f})$ for caspase processing or for cleavage of PARP (g), XIAP (h) and p23 (i). The Abs to caspase- 6 and -2 were from Cell Signaling Technology and Santa Cruz, respectively. The asterisk represents a nonspecific band

inhibiting to some degree the activity of apoptosomeassociated caspase-9 (Figure 1a, compare lanes 3-5 with lanes 6-9), whereas both inhibitors blocked the appearance of caspase- 9 processed at D330 compatible with this being a caspase-3 cleavage site.

More pronounced were the differences in the ability of the inhibitors to affect caspase- 3 and -7 processing. In the presence of etoposide, caspase-7 was processed to yield its $\sim$ p19 large subunit. Although this processing was inhibited in a concentration-dependent manner by z-VAD.fmk and this was accompanied by an increase in the unprocessed zymogen, it was not significantly inhibited by $M-791$ (Figure 1b). Caspase-3 was also initially processed to its 
$\sim$ p20 form and then caspase-3 readily autoprocessed to remove its prodomain following cleavage at D9 and 28 to yield the $\mathrm{p} 19$ and fully processed 17 forms (Figure 1c, lane 2). The autoprocessing of caspase- 3 was inhibited in a concentrationdependent manner by both inhibitors (Figure 1c) compatible with both inhibitors being capable of inhibiting caspase-3 activity. However, more caspase- 3 was clearly processed to its p20 form in the presence of M-791 than z-VAD.fmk (Figure 1c compare lanes 6-9 with 3-5), compatible with z-VAD.fmk being a more potent inhibitor than M-791 of apoptosome-associated caspase- 9 activity.

In earlier studies, using dATP-activated cell lysates, caspase-3 was absolutely responsible for the processing of both caspase- 2 and -6 , and active caspase- 6 was then responsible for the cleavage of caspase- $8 .^{22}$ In etoposide-exposed Jurkat cells, caspase- 6 was almost completely processed to its cleaved $\sim$ p17 large subunit (Figure 1d, lane 2). This processing was almost entirely blocked by z-VAD.fmk, but was only partially inhibited by M-791 (Figure 1d, compare lanes 3-5 with lanes 6-9). Although the processing of caspase- 6 to its $\sim$ p17 large subunit was inhibited in a concentrationdependent manner by z-VAD.fmk, this was accompanied by a partial, but incomplete restoration of the unprocessed caspase-6 zymogen (Figure 1d, upper panel - lanes 2-5). By contrast, the etoposide-induced processing of caspase- 6 was largely unaffected by $M-791$, except possibly at the highest concentration of M-791 (Figure 1d, lanes 6-9). Using a second caspase- 6 antibody (from MBL, Nagoya, Japan), the inhibition of the $\sim$ p17 subunit was also accompanied by the appearance of the proform together with a p20 large subunit (Supplementary Figure 1). Taken together, these data raise the possibility that the processing of caspase- 6 in cells undergoing apoptosis by the intrinsic pathway may be mediated by another caspase(s) in addition to caspase-3, in contrast to dATPactivated cell lysates.

Marked differences were also observed in the processing of caspase-2 in the presence of the two inhibitors. Caspase-2 is initially processed between the large and small subunits at D316 to yield an p33 fragment, corresponding to the prodomain and the large subunit and an $\sim$ p14 small subunit followed by a further cleavage at D330 by caspase- 3 to remove a linker region and form the $\sim$ p12 small subunit (Figure 1e, lane 2). The antibody used in our initial study (Santa Cruz, CA, USA) was raised to the C-terminus and so recognized the unprocessed zymogen and the processed small subunit but not the processed large subunit (Figure 1e lane 2). However, a second antibody (Alexis, Nottingham, UK) recognized both the $\mathrm{p} 33$ subunit and, on longer exposure, the fully processed $\sim$ p19 large subunit (Supplementary Figure 1, lane 2). Both inhibitors completely blocked the removal of the linker region, thereby preventing the formation of the fully processed small subunit p12 (Figure 1e, lanes 3-9) and supporting the notion that this removal is mediated by caspase-3. The initial processing of caspase-2 to form its p14 small subunit was predominantly unaffected by $\mathrm{M}-791$ (Figure 1e, lanes 6-9), whereas z-VAD.fmk significantly inhibited caspase-2 processing (Figure 1e). As z-VAD.fmk is a poor inhibitor of both caspase-2 activity and its autoprocessing, ${ }^{27}$ our data suggests that caspase-2 is not responsible for its own processing. Thus, the initial processing of caspase- 2 is completely unaffected when caspase- 3 activity is completely blocked, supporting the hypothesis that the initial cleavage of caspase- 2 is mediated by a caspase other than caspase- 2 and -3 .

Marked differences were observed in the processing of caspase- 8 in the presence of both inhibitors (Figure 1f). After exposure to etoposide, caspase- 8 was processed to its p43/41 forms, which arise following cleavage between the large and small subunits (Figure 1f, lanes 1-2). This processing was almost completely inhibited by z-VAD.fmk even at the lowest concentration used (Figure 1f, lanes 2-5). By contrast, whereas the lowest concentration of M-791 caused a partial inhibition of the processing of caspase-8, this was not substantially increased by higher M-791 concentrations (Figure 1f, lanes 6-9). Taken together, these results support the notion that although caspase- 8 may be processed by caspase-3, when caspase- 3 is inhibited another caspase, other than caspase-3, may process caspase-8.

We also studied the effects of the inhibitors on caspase substrates. Poly(ADP-ribose) polymerase (PARP) is cleaved at a canonical caspase-3/-7 motif DEVD $\downarrow$ G. ${ }^{1,28}$ Although originally described as a caspase-3 substrate, it is now recognized to be also cleaved by caspase-7. ${ }^{18,29}$ As X-linked inhibitor of apoptosis protein (XIAP) is cleaved by caspase-3 but not caspase-7, ${ }^{18}$ it can partially distinguish their activities. Cleavage of PARP and XIAP was observed in Jurkat cells exposed to etoposide (Figure $1 \mathrm{~g}$ and $\mathrm{h}$, lane 2). Cleavage of PARP was inhibited by $z-V A D$.fmk but not M-791, which is compatible with $M-791$ being a selective inhibitor of caspase-3 but not -7 , and thus caspase-7 being responsible for the cleavage (Figure 1g, lanes 3-9). However, cleavage of XIAP was inhibited by both inhibitors as the cleavage is only mediated by caspase-3. Examination of the effects of the two inhibitors on the cleavage of p23, which may be a specific caspase-7 substrate, ${ }^{30}$ showed that all z-VAD.fmk concentrations completely inhibited its cleavage, whereas only higher M-791 concentrations caused a slight inhibition (Figure 1i, compare lanes 3-5 with 4-9). Thus, these data further support the selectivity of M-791 as a specific inhibitor of caspase-3 activity.

Taken together, these data strongly support the suggestion that the order of caspase processing in intact cells undergoing apoptosis differs from that using dATP-activation of cell lysates. Our initial results highlight that although caspase-2, - 6 and -8 may be processed by caspase- 3 , these caspases should be processed by another caspase(s), when caspase-3 is inhibited.

Caspase- 9 but not -8 is required for etoposide-induced processing of caspase-2 and -6 . As caspase- 9 and -8 are the apical caspases in the intrinsic and extrinsic pathways, respectively, we utilized the availability of Jurkat cells deficient in these caspases to assess their roles. Caspase- 9 deficient Jurkat cells (JMR) were completely resistant to etoposide-induced apoptosis and no caspase processing was observed (Figure 2, lane 2 and Supplementary Figure 2). However, apoptosis and caspase processing was restored when caspase-9 was reintroduced (Figure 2, lane 5 and Supplementary Figure 2), in agreement with earlier studies. ${ }^{12}$ Furthermore, after stimulation of JMR cells with an anti-Fas 


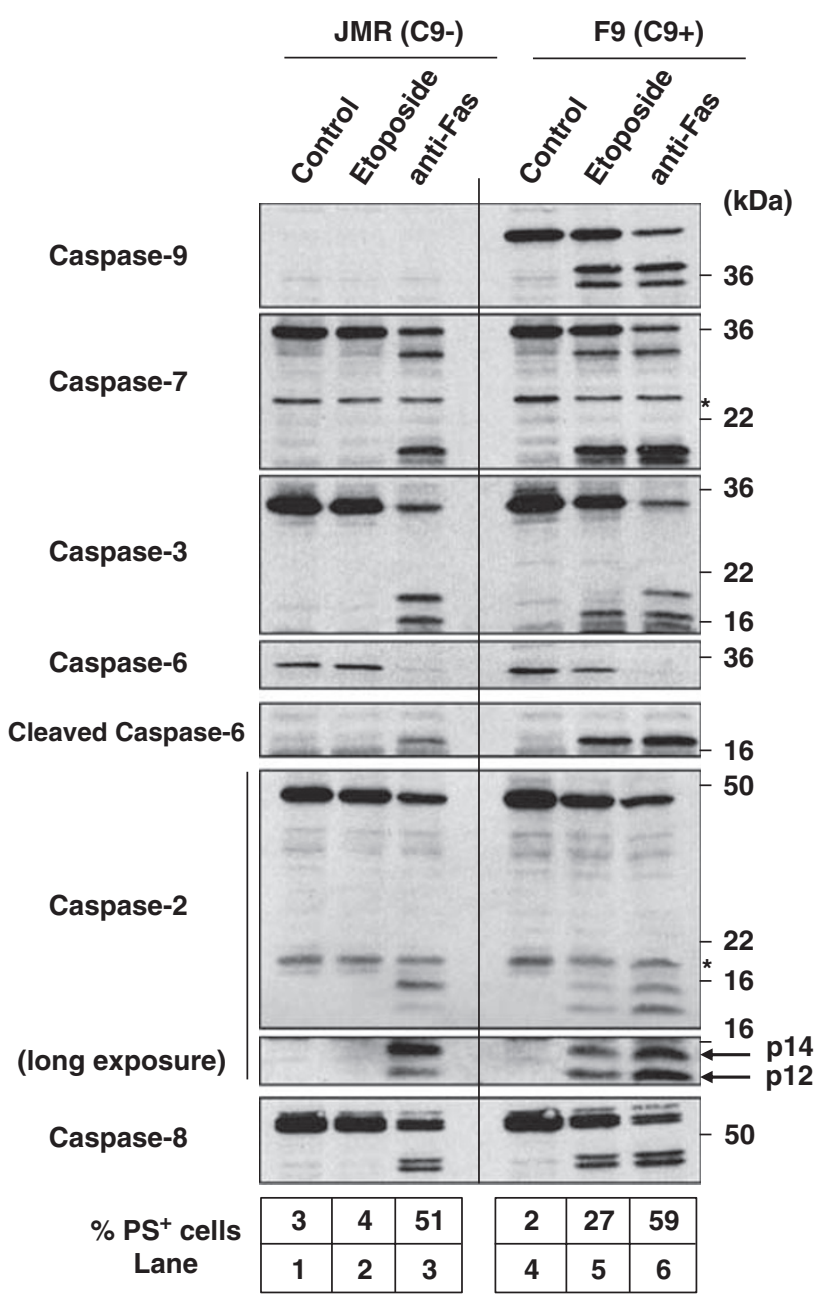

Figure 2 Caspase-2 and -6 are downstream of caspase-9 in etoposide-induced apoptosis. JMR (caspase-9 deficient) and F9 (caspase-9 stably expressing) Jurkat cells were exposed to either etoposide $(100 \mu \mathrm{M})$ (lanes 2 and 5$)$ or anti-Fas $(\mathrm{CH}-11$, $100 \mathrm{ng} / \mathrm{ml}$ ) (lanes 3 and 6 ) for $16 \mathrm{~h}$. Apoptosis was assessed by PS externalization as described in Materials and Methods and cells were analyzed by western blotting. The Abs to caspase- 6 and -2 were from Cell Signaling Technology and Santa Cruz, respectively. The asterisk represents a nonspecific band

antibody $(\mathrm{CH}-11)$, caspase-8 was activated together with all the downstream effector caspases (Figure 2, lane 3 ). In addition, caspase- 8 was also shown to be the apical caspase in the extrinsic pathway as no processing of any caspase was observed following anti-Fas stimulation of caspase-8 deficient Jurkat cells, whereas all caspases, including caspase-2 and -6 , were processed/activated after etoposide exposure (Supplementary Figure 3). These data show conclusively that processing of caspase- 2 and -6 is downstream of caspase- 8 and -9 in the extrinsic and intrinsic pathways in Jurkat cells, respectively.

Processing of caspase- 6 and -2 independent of caspase3. Our data with M-791 supported a role for caspases other than caspase- 3 in the processing of caspase- 2 and -6 . As activation of caspase- 9 leads to processing of caspase- 3 and -7 , we wished to ascertain the potential contribution of caspase-7 in the processing of caspase-2 and -6 under physiological conditions in intact cells. Therefore, we chose MCF-7 cells as they are deficient in caspase-3 (Supplementary Figure 4) and easier to transfect, ${ }^{31}$ thereby also excluding any possibility that M791 had not completely inhibited caspase-3 activity. Exposure of MCF-7 cells to etoposide or TRAIL resulted in the processing of caspase-9, $-7,-6,-2$ and -8 (Figure $3 a$ ). To induce rapid apoptosis through the intrinsic pathway, MCF-7 cells were also exposed to either staurosporine or MG132, a proteasome inhibitor, in the presence of $\mathrm{Mcl}-1$ small interference RNA (siRNA). This resulted in a rapid time-dependent processing of caspase-9, -7, -6, -2 and -8 (Supplementary Figures 5a and b). These data show conclusively that in cells undergoing apoptosis, by either the intrinsic or extrinsic pathway, both caspase- 2 and -6 can be processed by a caspase other than caspase-3. Furthermore, the processing of the caspases was also accompanied by the cleavage of lamin A/C (caspase- 6 substrate), p23 (caspase-7 substrate), PARP (caspase-3, 6 and -7 substrate) and Bid (caspase-3 and -8 substrate) ${ }^{19}$ (Figure $3 b$, lanes 2 and 3 and Supplementary Figure 5). Both staurosporine and MG132 induced a rapid processing of caspase-9, which preceded the processing/cleavage of caspase-7 and -2, p23 and PARP, as well as the initial cleavage of caspase- 6 to its p20 form (Supplementary Figure 5). At later times, the p20 form of caspase- 6 is further processed to a p17 form and the appearance of this seems to correspond with the processing/ cleavage of caspase- 8 and lamin $A / C$ and this is most marked with MG132 (Supplementary Figure 5).

Processing of caspase-2 and -6 is downstream of caspase-7. To more directly ascertain the possible role of caspase-7 in the processing of caspase-2 and -6 in MCF-7 cells, we used siRNA for caspase-7. In these studies, staurosporine was used as it induces apoptosis more rapidly and extensively than etoposide. Staurosporine-induced apoptosis was accompanied by processing of caspase-9, -7, -2, -6 and -8 (Figure 4). Knockdown of caspase-7 was effective as judged by short exposure of the western blot although longer exposure showed some caspase-7 was still present (Figure 4, compare lanes 1 and 2). Following exposure to staurosporine, this knockdown resulted in extensive but not complete inhibition of apoptosis accompanied by marked inhibition of the processing of caspase-2, -6 and -8 and a smaller inhibition of the processing of caspase-9 (Figure 4, compare lanes 3 and 4). The residual apoptosis and caspase processing was most probably because of some residual caspase-7, as complete inhibition was observed with z-VAD.fmk (Figure 4, lane 5). Knockdown of caspase-7 resulted in almost complete inhibition of cleavage of lamin A/C, p23 and PARP (Figure 4) in staurosporine-exposed cells. These data show that processing of caspase-7 is upstream of the processing of caspase-2, -6 and -8 .

Caspase-7 processes caspase-6, which then processes caspase-8. We next explored the role of caspase- 6 in the intrinsic pathway. Exposure of MCF-7 cells to siRNA for caspase- 6 resulted in a specific but incomplete knockdown of caspase-6 without affecting other caspases (Figure 5, compare lanes 1 and 3 ). Exposure of these cells to 


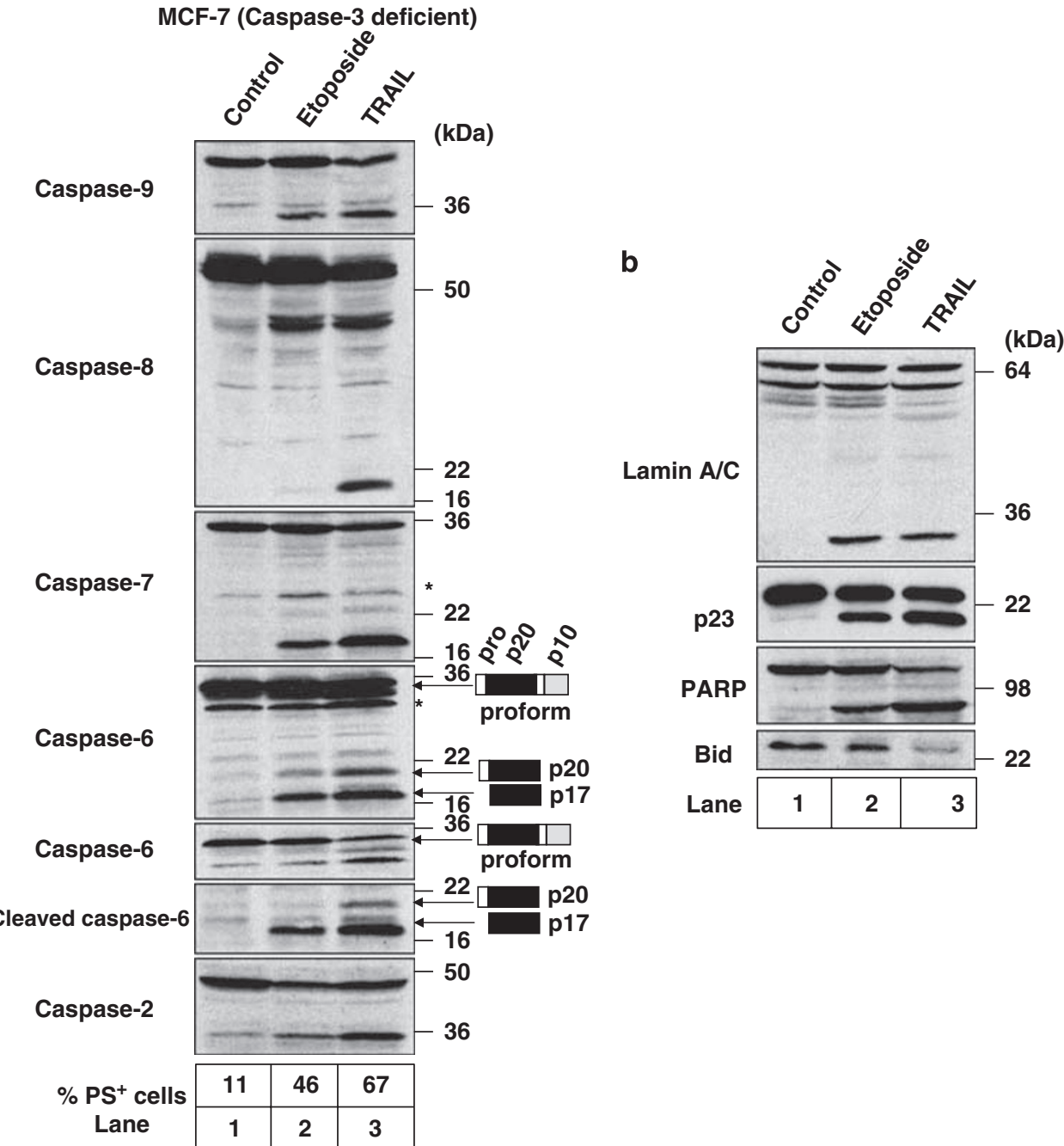

Figure 3 Caspase-2 and -6 can be processed in a caspase-3 independent manner. (a) MCF-7 cells, which are caspase-3 deficient, were exposed to either etoposide $(200 \mu \mathrm{M})$ (lane 2) or TRAIL (1 $\mu \mathrm{g} / \mathrm{ml}$ ) (lane 3 ) for 24 or $10 \mathrm{~h}$, respectively. (b) Apoptosis was assessed by PS externalization as described in Materials and Methods and cells were analyzed by western blotting for caspase processing and substrate cleavage. The Ab to caspase-2 was from Alexis Corporation. Two different Abs to caspase-6 were used, in the upper panel the Ab was from MBL and in the lower two panels from Cell Signaling Technology. The asterisk represents a nonspecific band

staurosporine resulted in a small decrease in apoptosis (Figure 5, lanes 2 and 4). Although the caspase-6 knockdown was incomplete, it resulted in an almost complete inhibition of the processing of caspase- 8 but not of caspase- 9 or -7 , together with a small decrease in caspase-2 processing (Figure 5 lanes 2 and 4). The small decrease in caspase-2 processing observed is suggestive of a minor involvement of caspase- 6 in a feed-forward amplification loop. Furthermore, exposure of MCF-7 cells with caspase- 6 knockdown to staurosporine resulted in complete inhibition of lamin $A / C$ cleavage, without any effect on p23 or PARP cleavage (Figure 5). Similar results were obtained using different caspase-6 siRNA oligonucleotides (Supplementary Figure 6). Taken together, these data are compatible with caspase-9 initially cleaving caspase-7, which then processes and activates caspase-6, which then cleaves caspase-8 and lamin A/C.

Caspase- 6 is a cytoplasmic protein in MCF-7 cells. To investigate where in the cell the processing of caspase- 6 by caspase-7 took place, nuclear and cytoplasmic fractions were prepared (Figure 6a). The purity of the nuclear and cytoplasmic fractions was verified using nuclear (Histone $\mathrm{H} 4$, lamin A/C and PARP) and cytoplasmic (tubulin) markers (Figure 6a). Although all the caspases were found exclusively in the cytoplasmic fraction, we cannot exclude that there may have been some leakage of nuclear caspases during the fractionation process. These data suggest that the initial caspase- 6 processing occurs primarily within the cytoplasm and active caspase- 6 then translocates to the nucleus, where it cleaves nuclear substrates such as lamin A/C.

\section{Discussion}

Using dATP-activated lysates to mimic activation of the intrinsic pathway, a hierarchy of caspase activation was established in which caspase-9, as the apical caspase, directly activates caspase-3 and -7 . Active caspase-3, but 


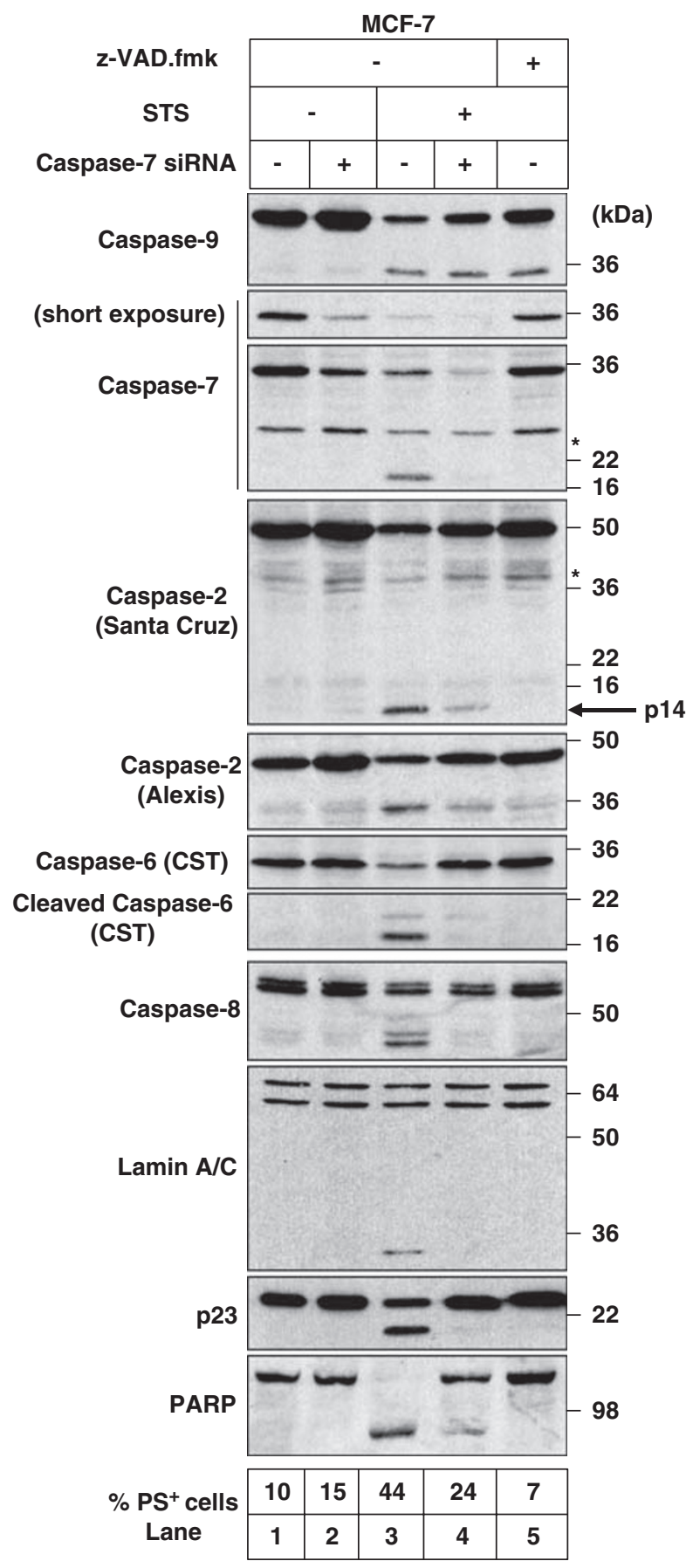

Figure 4 Caspase-2 and -6 are processed downstream of caspase-7 in staurosporine-induced apoptosis in MCF-7 cells. MCF-7 cells were transfected with either control (lanes 1, 3 and 5) or caspase-7 siRNA (lanes 2 and 4) oligonucleotides for $36 \mathrm{~h}$. Cells were then exposed to staurosporine (STS, $1.5 \mu \mathrm{M}$ ) for $12 \mathrm{~h}$ in the absence (lanes 1-4) or presence (lane 5) of z-VAD.fmk (25 $\mu \mathrm{M})$. Apoptosis was assessed by PS externalization as described in Materials and Methods and cells were analyzed by western blotting for caspase processing or substrate cleavage. Two different Abs to caspase-2 were used, in the upper panel the Ab was from Santa Cruz and in the lower panel from Alexis Corporation. The asterisk represents a nonspecific band

not -7 , then activates caspase -6 and -2 and active caspase- 6 cleaves caspase-8. ${ }^{18,22}$ Although this model has generally been assumed to be the paradigm for caspase activation in

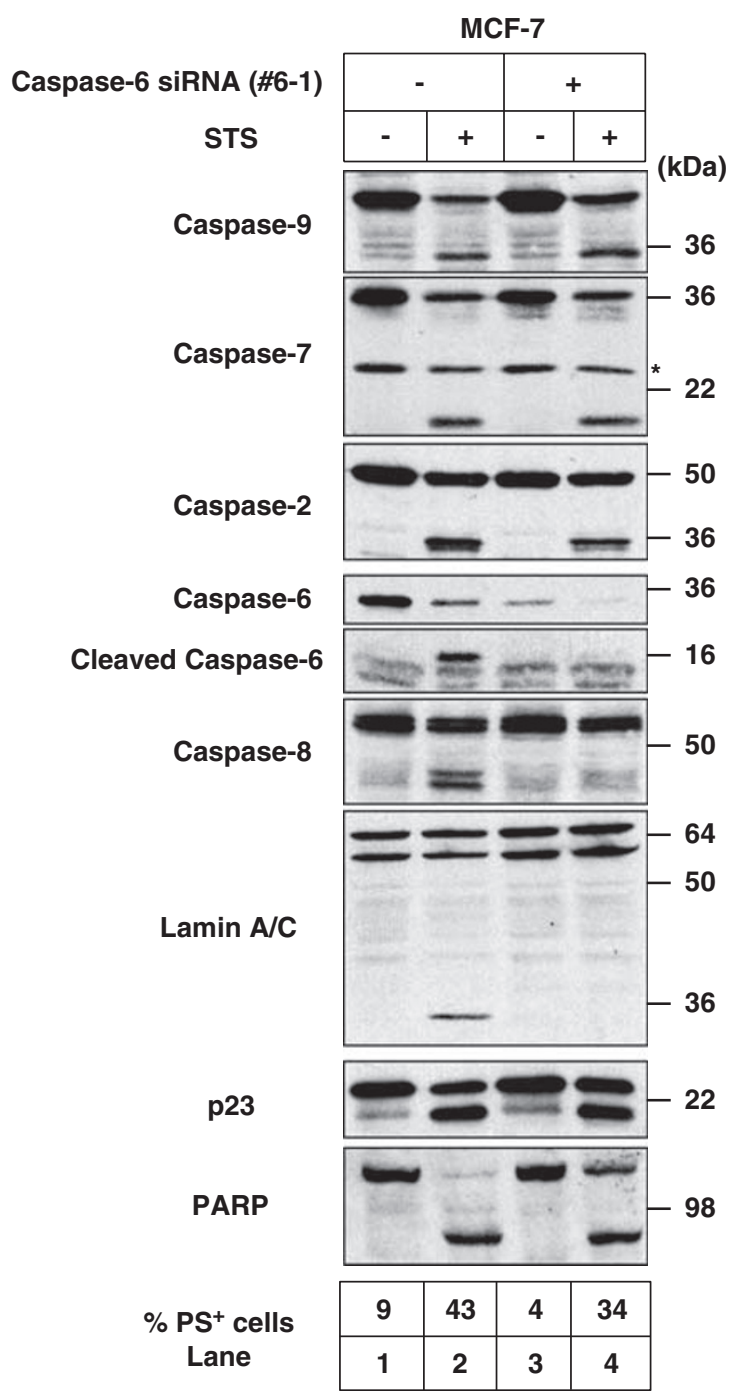

Figure 5 Caspase-2 can be processed by caspase-7 but not caspase- 6 in staurosporine-induced apoptosis in MCF-7 cells. MCF-7 cells were transfected with either control (lanes 1-2) or caspase-6 (lanes 3-4) oligonucleotides for $36 \mathrm{~h}$. Cells were then exposed to staurosporine (STS, $1.5 \mu \mathrm{M}$ ) (lanes 2 and 4 ) for $12 \mathrm{~h}$ and apoptosis assessed by PS externalization as described in Materials and Methods. Cells were analyzed by western blotting for caspase processing and substrate cleavage. The Abs to caspase- 6 and -2 were from Cell Signaling Technology and Alexis Corporation, respectively. The asterisk represents a nonspecific band

cells undergoing apoptosis, ${ }^{23}$ the phenotype of knockout mice was not entirely consistent with this model, particularly in relation to redundancy/compensation between caspase-3 and caspase-7. ${ }^{24}$ Furthermore, recombinant caspase-7 can also cleave both caspase-2 and $-6 .{ }^{15}$ To resolve this discrepancy, we examined the hierarchical ordering of caspases in intact cells after activation of the intrinsic pathway under physiological conditions using M-791, a caspase-3 specific inhibitor and siRNA of caspases in caspase-3deficient MCF-7 cells. After initial mitochondrial perturbation, activated caspase- 9 activates both effector caspase -3 and -7 . In contrast to the established model using dATP-activated cell lysates, caspase-7, in addition to caspase-3, can directly process caspase-6, and subsequently the activated caspase- 
a

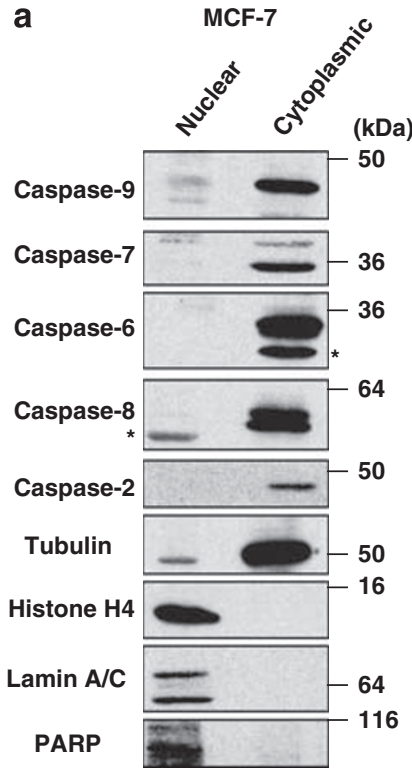

b

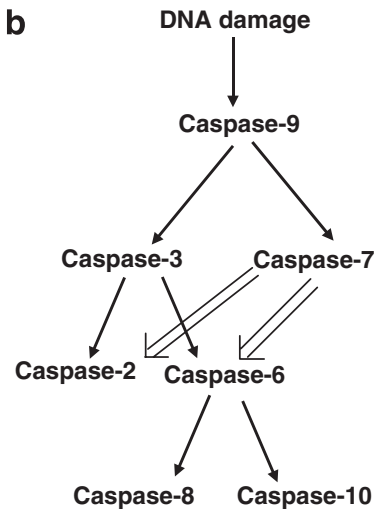

Figure 6 Hierarchical ordering of caspases in cells undergoing apoptosis activated by the intrinsic pathway. (a) Nuclear (lane 1) and cytoplasmic (lane 2) fractions were prepared by differential centrifugation from MCF-7 cells as described in Materials and Methods. Protein samples from each fraction were analyzed by western blotting. The Abs to caspase- 6 and -2 were from MBL and Alexis Corporation, respectively. The unprocessed caspase zymogens are present exclusively in the cytoplasm. The asterisk represents a nonspecific band. (b) Schematic representation of hierarchical ordering of caspases in cells undergoing apoptosis activated by the intrinsic pathway. The double arrows represent new pathways of activation shown in this study

6 can directly process caspase-8 (Figure 6b). The cleavage of caspase- 6 by caspase- 7 could be required for completion of nuclear apoptotic events, including chromatin condensation and apoptotic body formation. ${ }^{20}$ Such caspase-7 mediated caspase- 6 activation may be particularly important in cells deficient in caspase-3, including some breast tumors and neuroblastoma. ${ }^{32,33}$

Although many inhibitors of caspases have been described relatively few are specific. ${ }^{34,35}$ Interestingly, in vitro $\mathrm{M}-791$ inhibits recombinant caspase- 3 specifically with an $\mathrm{IC}_{50}=$ $8 \mathrm{nM}$ at least 30 -fold more potent than all other caspases. ${ }^{25} \mathrm{In}$ this study, we have examined the specificity of M-791 to inhibit caspases in cells, as assessed by caspase processing and substrate cleavage (Figure 1). M-791 caused a concentrationdependent inhibition of the autoprocessing of caspase $-3^{36}$ and the cleavage of two caspase- 3 substrates, XIAP and cleavage of caspase-9 at D330. ${ }^{18,37}$ Furthermore, M-791 inhibited neither the processing of caspase- $6,-7$ and -8 nor the initial processing of caspase- 9 and -2 . However, it did inhibit the secondary processing of caspase- 9 and caspase-2 to their p35 and p12 forms, respectively, (Figures 1a and e) indicating that these secondary cleavages are most likely mediated by caspase-3. The cleavage of PARP was not inhibited at all concentrations of M-791 studied (Figure 1g, lanes 6-9), in contrast to z-VAD.fmk $(10 \mu \mathrm{M})$, which completely inhibited PARP cleavage (Figure 1g). As PARP is primarily a substrate for caspase- 3 and -7 and also a minor substrate for caspase- $6,{ }^{18}$ our results suggest that M-791 did

not significantly inhibit the activity of caspase- 6 and/or -7 in intact cells undergoing apoptosis. Furthermore, cleavage of p23, a caspase-7 specific substrate, was only partially inhibited by high M-791 concentrations (Figure 1i). Taken together, these data strongly suggest that in intact cells M-791 is a highly selective and potent inhibitor of caspase- 3 and could be a valuable tool to identify specific functions of caspase-3.

Where caspase-2 is processed in the caspase cascade and its significance are controversial. Using cell lysates and recombinant proteins, caspase-2 was shown to either autoprocess or to be processed by caspase- $3,-8$, or possibly -7 to yield a large (p33) and small (p14) subunit. The small subunit was subsequently processed by caspase-3 at D330 to yield a p12 form. ${ }^{15,18,22,37,38}$ In agreement with an earlier study, ${ }^{12}$ caspase-2 was not processed in caspase-9 deficient Jurkat cells (Figure 2). Both z-VAD.fmk and caspase-7 but not caspase-6 siRNA (Figures 4 and 5) inhibited the processing of caspase-2. Caspase-2 is cleaved to p33 and p14, but not p12 in MCF-7 cells (Figure 4). Taken together, these data show that, in cells, caspase-7, in addition to caspase-3, may act upstream of caspase-2 and be responsible for its initial processing either directly or indirectly, although this also may be cell type dependent. ${ }^{14}$ Furthermore, the secondary cleavage of the 114 small subunit of caspase-2 to its $\mathrm{p}^{12}$ form is mediated by caspase-3 but not caspase- 7 .

Using recombinant procaspase- 3 and -6 , it was shown that caspase- 6 was cleaved at three sites. Initial cleavage occurred predominantly at DVVD ${ }^{179} \downarrow N$, a canonical caspase3 cleavage site, between the large (p20 form) and small subunits, followed possibly by cleavage at D193 to remove a linker region on the small subunit. ${ }^{37}$ Removal of the prodomain by cleavage of the large subunit at D23 resulted in further processing to form a p17 large subunit. The importance of removal of prodomain for the activity of caspase- 6 in cells has not been elucidated. The nature of the processing of caspase- 6 in intact cells undergoing apoptosis observed in our study (Figure 3 and Supplementary Figure 5) is generally compatible with these earlier in vitro results. In MCF-7 cells induction of apoptosis resulted in a time-dependent loss of the proform of caspase- 6 that was accompanied by the initial appearance of a p20 form followed by removal of its prodomain to form a p17 fragment (Supplementary Figure 5). Interestingly, the kinetics of cleavage of lamin $A / C$ and caspase-8 appeared similar to the appearance of $p 17$ form of caspase-6 (Supplementary Figure 5) raising the possibility that most of the lamin $A / C$ cleavage by caspase- 6 was because of its p17/p11 rather than the p20/p11 form. Removal of the prodomain of caspase- 7 is required for its efficient activation in vivo, possibly because the highly charged $\mathrm{N}$-terminal prodomain results in the physical sequestration of caspase-7 from its upstream caspase ${ }^{39}$ To our knowledge there has only been one earlier study to investigate the importance of the removal of prodomain of caspase- 6 in cells. ${ }^{40}$ They showed that the removal of the prodomain of caspase- 6 was required both for caspase-8 processing and also for serum-depletion-mediated apoptosis. Taken together with our data, these results raise the possibility that removal of the prodomain of caspase- 6 is required for efficient catalytic activity as observed by its ability to cleave both lamin A/C 
and caspase-8 (Supplementary Figure 5). Removal of the Nterminal 23 amino acids may also possibly facilitate translocation of active caspase- 6 to the nucleus by increasing the accessibility of a potential nuclear localization signal $\left(\mathrm{HRRR}^{44}\right)$ or affect its three-dimensional structure required for substrate interaction. In this regard, the $\mathrm{N}$-terminal 30 amino acids of procaspase- 6 are predicted to be disordered, using regional order neural network (RONN) software for the detection of natively disordered region in proteins. ${ }^{41}$ Another possibility is removal of the prodomain relieves caspase- 6 from an endogenous inhibitor of caspases, although there is no evidence that caspase- 6 interacts with XIAP, c-IAP1 or caspase- $2 .{ }^{42} \mathrm{How}-$ ever, other possible anti-apoptotic proteins could associate with the prodomain of caspase- 6 in an analogous manner to the association of $\mathrm{Hsp} 27$ with the prodomain of caspase-3, which results in the inhibition of the autocatalytic processing of caspase- 3 from its $p 20$ to its p17 form that is required for its efficient processing and activation. ${ }^{43}$

In summary, in contrast to the established model using dATP-activated cell lysates, caspase-7 in addition to caspase- 3 can directly process caspase- 6 , and subsequently the processed caspase- 6 can directly process caspase-8.

\section{Materials and Methods}

Cell lines and culture. Jurkat E6.1 (European Collection of Animal Cell Cultures), Jurkat A3 and caspase-8 deficient cells (Dr. J Blenis, Harvard Medical School, Boston, MA, USA), caspase-9 deficient Jurkat (clone JMR) and caspase-9 stably transfected Jurkat JMR cells (clone F9) ${ }^{12,44}$ (Professor Ingo Schmitz, Dusseldorf, Germany) and MCF-7-Fas (MCF-7) cells (Dr. M Jattella, Danish Cancer Society Research Center, Copenhagen, Denmark) were cultured in RPMI 1640 medium supplemented with 10\% FBS and 5\% Glutamax.

Reagents. Media and serum were from Life Technologies Inc., (Paisley, UK). Antibodies were sourced as follows: rabbit cleaved caspase-9 (D315, \#9505), rabbit cleaved caspase-9 (D330, \#9501), rabbit caspase-6 (\#9762), rabbit cleaved caspase-6 (D162, \#9761), rabbit histone H4 (\#2592) and rabbit lamin A/C (\#2032) were from Cell Signaling Technology (Beverly, MA, USA). The mouse caspase-6 mAb (M070-3) and mouse caspase-9 (M054-3) mAb were from MBL. The rabbit caspase-2 (C-20; sc-625) was from Santa Cruz. The rat caspase-2 mAb (11B4; ALX-804-356-C100) and mouse anti-PARP mAb (C2-10; ALX-804-210-R050) were from Alexis (Nottingham, UK). The mouse anti-p23 mAb (MA3-414) was from Affinity Bioreagents (Golden, CA, USA). The mouse anti-XIAP mAb, mouse anti-tubulin $m A b$, rabbit Bid $A b$ and caspase- $7,-3$ and -8 antibodies were from sources as described earlier. ${ }^{7,45}$ The anti-Fas $\mathrm{Ab}(\mathrm{CH}-11)$ was from Upstate (Lake Placid, NY, USA). MG132 was from Calbiochem (La Jolla, CA, USA). The human recombinant TRAlL was described earlier. ${ }^{45}$ The caspase-3 specific inhibitor, Cilastatin (M-791), was a kind gift from Dr. H Mehmet (Merck \& Co. Inc., Rahway, $\mathrm{NJ}$, USA).$^{25}$ Other chemicals, unless stated, were from Sigma (Poole, UK).

Quantification of apoptosis and western blot analysis. Apoptosis was quantified either by loss in mitochondrial membrane potential or by phosphatidylserine (PS) externalization in the presence of propidium iodide and samples for western blotting were assessed as described earlier. ${ }^{45}$ Before gel electrophoresis, protein levels were determined by the Bradford method and equal amounts of protein were loaded. This was confirmed by Ponceau staining after western blotting.

Small interference RNA of caspase- 6 and $\mathbf{- 7}$. MCF-7 cells ( $\sim 40000$ cells per well in 6-well plates) were grown for $24 \mathrm{~h}$, followed by transfection with siRNA oligonucleotides (Mcl-1 $(25 \mathrm{nM})$, caspase- $6(30 \mathrm{nM})$ and caspase-7 $(45 \mathrm{nM})$ ) using Hyperfect (Qiagen, Crawley, West Sussex, UK). Following transfection for $36 \mathrm{~h}$, cells were then exposed to MG132 $(1 \mu \mathrm{M})$ (for Mcl-1 siRNA) for $6 \mathrm{~h}$ or staurosporine $(1.5 \mu \mathrm{M})$ (for caspase- 6 and -7 siRNA) for $12 \mathrm{~h}$, respectively. The Mcl- 1 siRNA was the same as described earlier. ${ }^{46}$ The caspase- 6 siRNA (ID: 4131 for \#6-1 and 3942 for \#6-3) and untargeted siRNA (ID 4635) oligonucleotides were from Ambion (Austin, TX, USA). The caspase-7 siRNA (SMART pool; ID: L-00440700-0005) oligonucleotides were from Thermo Scientific (Waltham, MA, USA).

Preparation of nuclear and cytoplasmic fraction. Nuclear and cytoplasmic fractions from MCF-7 cells $\left(5 \times 10^{6}\right.$ cells) were prepared as described earlier. ${ }^{47}$

Acknowledgements. We thank Profs I Schmitz and K Schulze-Osthoff for supplying caspase-9 deficient Jurkat (clone JMR) and caspase-9 stably transfected Jurkat JMR cells (clone F9). We thank Dr. Huseyin Mehmet (Merck \& Co.) for supplying M-791. We also thank Drs. Alison Faragher and Davina Twiddy (MRC Toxicology Unit, Leicester, UK) for discussion of caspase-6 siRNA. This work was supported by the Medical Research Council.

1. Cohen GM. Caspases: the executioners of apoptosis. Biochem J 1997; 326: 1-16.

2. Earnshaw WC, Martins LM, Kaufmann SH. Mammalian caspases: structure, activation, substrates, and functions during apoptosis. Annu Rev Biochem 1999; 68: 383-424.

3. Fuentes-Prior $P$, Salvesen GS. The protein structures that shape caspase activity, specificity, activation and inhibition. Biochem J 2004; 384: 201-232.

4. Thornberry NA, Lazebnik Y. Caspases: enemies within. Science 1998; 281: 1312-1316.

5. Bratton SB, MacFarlane M, Cain K, Cohen GM. Protein complexes activate distinct caspase cascades in death receptor and stress-induced apoptosis. Exp Cell Res 2000; 256: 27-33.

6. Danial NN, Korsmeyer SJ. Cell death: critical control points. Cell 2004; 116: 205-219.

7. Sun XM, MacFarlane M, Zhuang J, Wolf BB, Green DR, Cohen GM. Distinct caspase cascades are initiated in receptor-mediated and chemical-induced apoptosis. J Biol Chem 1999; 274: 5053-5060.

8. Bratton SB, Walker G, Srinivasula SM, Sun XM, Butterworth M, Alnemri ES et al. Recruitment, activation and retention of caspases- 9 and -3 by Apaf- 1 apoptosome and associated XIAP complexes. EMBO J 2001; 20: 998-1009.

9. Jiang X, Wang X. Cytochrome c-mediated apoptosis. Annu Rev Biochem 2004; 73 87-106.

10. Li P, Nijhawan D, Budihardjo I, Srinivasula SM, Ahmad M, Alnemri ES et al. Cytochrome $c$ and dATP-dependent formation of Apaf-1/caspase-9 complex initiates an apoptotic protease cascade. Cell 1997; 91: 479-489.

11. Lassus $P$, Opitz-Araya X, Lazebnik Y. Requirement for caspase-2 in stress-induced apoptosis before mitochondrial permeabilization. Science 2002; 297: 1352-1354.

12. Samraj AK, Sohn D, Schulze-Osthoff K, Schmitz I. Loss of caspase-9 reveals its essential role for caspase-2 activation and mitochondrial membrane depolarization. Mol Biol Cell 2007; 18: 84-93.

13. Tinel A, Tschopp J. The PIDDosome, a protein complex implicated in activation of caspase2 in response to genotoxic stress. Science 2004; 304: 843-846.

14. Zhivotovsky B, Orrenius S. Caspase-2 function in response to DNA damage. Biochem Biophys Res Commun 2005; 331: 859-867.

15. Van de Craen M, Declercq W, Van den brande I, Fiers W, Vandenabeele P. The proteolytic procaspase activation network: an in vitro analysis. Cell Death Differ 1999; 6: 1117-1124.

16. Galande S, Dickinson LA, Mian IS, Sikorska M, Kohwi-Shigematsu T. SATB1 cleavage by caspase 6 disrupts PDZ domain-mediated dimerization, causing detachment from chromatin early in T-cell apoptosis. Mol Cell Biol 2001; 21: 5591-5604.

17. Hirata H, Takahashi A, Kobayashi S, Yonehara S, Sawai H, Okazaki T et al. Caspases are activated in a branched protease cascade and control distinct downstream processes in Fas-induced apoptosis. J Exp Med 1998; 187: 587-600.

18. Slee EA, Adrain C, Martin SJ. Executioner caspase-3, -6, and -7 perform distinct, non-redundant roles during the demolition phase of apoptosis. J Biol Chem 2001; 276: 7320-7326.

19. Takahashi A, Alnemri ES, Lazebnik YA, Fernandes-Alnemri T, Litwack G, Moir RD et al. Cleavage of lamin A by Mch2 alpha but not CPP32: multiple interleukin 1 beta-converting enzyme-related proteases with distinct substrate recognition properties are active in apoptosis. Proc Natl Acad Sci USA 1996; 93: 8395-8400.

20. Ruchaud S, Korfali N, Villa P, Kottke TJ, Dingwall C, Kaufmann SH et al. Caspase-6 gene disruption reveals a requirement for lamin A cleavage in apoptotic chromatin condensation. EMBO J 2002; 21: 1967-1977.

21. Stennicke HR, Deveraux QL, Humke EW, Reed JC, Dixit VM, Salvesen GS. Caspase-9 can be activated without proteolytic processing. J Biol Chem 1999; 274: 8359-8362.

22. Slee EA, Harte MT, Kluck RM, Wolf BB, Casiano CA, Newmeyer DD et al. Ordering the cytochrome c-initiated caspase cascade: hierarchical activation of caspases-2, $-3,-6,-7$, -8 , and -10 in a caspase-9-dependent manner. J Cell Biol 1999; 144: 281-292.

23. Taylor RC, Cullen SP, Martin SJ. Apoptosis: controlled demolition at the cellular level. Nat Rev Mol Cell Biol 2008; 9: 231-241.

24. Lakhani SA, Masud A, Kuida K, Porter Jr GA, Booth CJ, Mehal WZ et al. Caspases 3 and 7 : key mediators of mitochondrial events of apoptosis. Science 2006; 311: 847-851.

25. Hotchkiss RS, Chang KC, Swanson PE, Tinsley KW, Hui JJ, Klender P et al. Caspase inhibitors improve survival in sepsis: a critical role of the lymphocyte. Nat Immunol 2000; 1 496-501. 
26. Srinivasula SM, Hegde R, Saleh A, Datta P, Shiozaki E, Chai J et al. A conserved XIAPinteraction motif in caspase- 9 and Smac/DIABLO regulates caspase activity and apoptosis. Nature 2001; 410: 112-116.

27. Garcia-Calvo M, Peterson EP, Leiting B, Ruel R, Nicholson DW, Thornberry NA. Inhibition of human caspases by peptide-based and macromolecular inhibitors. J Biol Chem 1998 273: 32608-32613.

28. Lazebnik YA, Kaufmann SH, Desnoyers S, Poirier GG, Earnshaw WC. Cleavage of poly(ADP-ribose) polymerase by a proteinase with properties like ICE. Nature 1994; 371 346-347.

29. Germain M, Affar EB, D'Amours D, Dixit VM, Salvesen GS, Poirier GG. Cleavage of automodified poly(ADP-ribose) polymerase during apoptosis. Evidence for involvement of caspase-7. J Biol Chem 1999; 274: 28379-28384.

30. Walsh JG, Cullen SP, Sheridan C, Luthi AU, Gerner C, Martin SJ. Executioner caspase-3 and caspase-7 are functionally distinct proteases. Proc Natl Acad Sci USA 2008; 105 : 12815-12819.

31. Janicke RU, Sprengart ML, Wati MR, Porter AG. Caspase-3 is required for DNA fragmentation and morphological changes associated with apoptosis. J Biol Chem 1998; 273: 9357-9360.

32. Devarajan E, Sahin AA, Chen JS, Krishnamurthy RR, Aggarwal N, Brun AM et al. Downregulation of caspase 3 in breast cancer: a possible mechanism for chemoresistance. Oncogene 2002; 21: 8843-8851

33. Iolascon A, Borriello A, Giordani L, Cucciolla V, Moretti A, Monno F et al. Caspase 3 and 8 deficiency in human neuroblastoma. Cancer Genet Cytogenet 2003; 146: 41-47.

34. McStay GP, Salvesen GS, Green DR. Overlapping cleavage motif selectivity of caspases: implications for analysis of apoptotic pathways. Cell Death Differ 2008; 15: 322-331.

35. Villa P, Kaufmann SH, Earnshaw WC. Caspases and caspase inhibitors. Trends Biochem Sci 1997; 22: 388-393.

36. Han Z, Hendrickson EA, Bremner TA, Wyche JH. A sequential two-step mechanism for the production of the mature p17:p12 form of caspase-3 in vitro. J Biol Chem 1997; 272: 13432-13436.
37. Srinivasula SM, Fernandes-Alnemri T, Zangrilli J, Robertson N, Armstrong RC, Wang L et al. The Ced-3/interleukin 1beta converting enzyme-like homolog Mch6 and the lamincleaving enzyme Mch2alpha are substrates for the apoptotic mediator CPP32. J Biol Chem 1996; 271: 27099-27106.

38. Baliga BC, Read SH, Kumar S. The biochemical mechanism of caspase-2 activation. Cell Death Differ 2004; 11: 1234-1241.

39. Denault JB, Salvesen GS. Human caspase-7 activity and regulation by its N-terminal peptide. J Biol Chem 2003; 278: 34042-34050.

40. Cowling V, Downward J. Caspase- 6 is the direct activator of caspase- 8 in the cytochrome c-induced apoptosis pathway: absolute requirement for removal of caspase- 6 prodomain. Cell Death Differ 2002; 9: 1046-1056.

41. Yang ZR, Thomson R, McNeil P, Esnouf RM. RONN: the bio-basis function neural network technique applied to the detection of natively disordered regions in proteins. Bioinformatics 2005; 21: 3369-3376.

42. Roy N, Deveraux QL, Takahashi R, Salvesen GS, Reed JC. The C-IAP-1 and C-IAP-2 proteins are direct inhibitors of specific caspases. EMBO J 1997; 16: 6914-6925.

43. Voss OH, Batra S, Kolattukudy SJ, Gonzalez-Mejia ME, Smith JB, Doseff Al. Binding of caspase-3 prodomain to heat shock protein 27 regulates monocyte apoptosis by inhibiting caspase-3 proteolytic activation. J Biol Chem 2007; 282: 25088-25099.

44. Samraj AK, Keil E, Ueffing N, Schulze-Osthoff K, Schmitz I. Loss of caspase-9 provides genetic evidence for the type I/II concept of CD95-mediated apoptosis. J Biol Chem 2006; 281: 29652-29659.

45. Inoue S, MacFarlane M, Harper N, Wheat LM, Dyer MJ, Cohen GM. Histone deacetylase inhibitors potentiate TNF-related apoptosis-inducing ligand (TRAIL)-induced apoptosis in lymphoid malignancies. Cell Death Differ 2004; 11 (Suppl 2): S193-S206.

46. Inoue S, Walewska R, Dyer MJ, Cohen GM. Downregulation of Mcl-1 potentiates HDACi-mediated apoptosis in leukemic cells. Leukemia 2008; 22: 819-825.

47. Faragher AJ, Sun XM, Butterworth M, Harper N, Mulheran M, Ruchaud S et al. Death receptor-induced apoptosis reveals a novel interplay between the chromosomal passenger complex and CENP-C during interphase. Mol Biol Cell 2007; 18: 1337-1347.

Supplementary information accompanies the paper on Cell Death and Differentiation website (http://www.nature.com/cdd) 